\title{
Accurate Spectrophotometric Method for Semitransparent Metallic Thin-film Index Determination Using Interference Enhancement
}

Riley Shurvinton ( $\sim$ clshurv@gmail.com )

Institut Fresnel Marseille https://orcid.org/0000-0001-5122-0693

Fabien Lemarchand

Institut Fresnel Marseille

Antonin Moreau

Institut Fresnel Marseille

Julien Lumeau

Institut Fresnel Marseille

\section{Research}

Keywords: spectrophotometry, index determination, absorbing layers, titanium

Posted Date: July 23rd, 2021

DOI: https://doi.org/10.21203/rs.3.rs-660206/v1

License: (c) (i) This work is licensed under a Creative Commons Attribution 4.0 International License. Read Full License 


\title{
Accurate spectrophotometric method for semitransparent metallic thin-film index determination using interference enhancement
}

\author{
Riley Shurvinton*, Fabien Lemarchand, Antonin Moreau and Julien Lumeau
}

\begin{abstract}
An accurate spectrophotometric method to determine the refractive index of a semitransparent metallic thin film is presented. This method relies on interference enhancement of the measured spectra, employing an opaque substrate with a dielectric spacer layer beneath the absorbing layer of interest to create interference fringes. The resulting spectral oscillations of the stack are highly sensitive to the properties of the top absorbing layer, allowing precise determination of the refractive index via fitting. The performance of this method is verified using simulations in comparison to the typical method of depositing the absorbing thin film directly onto a transparent substrate. An experimental demonstration is made for titanium thin films over the visible range (370-835 nm). The refractive index of these films is extracted from experimental data using a combination of the Modified Drude and Forouhi-Bloomer models. This method showed high repeatability and accuracy, and is verified for Ti films between 6-70 nm thickness.
\end{abstract}

Keywords: spectrophotometry; index determination; absorbing layers; titanium

\section{Introduction}

Precise determination of the refractive index of materials is of paramount importance in the field of optical coatings. To accurately control the optical properties of thin-film coatings, it is imperative to know the refractive index of these materials to a high degree of accuracy. However, the refractive index dispersion of a given material when deposited as a thin-film often differs from the bulk value reported in literature, and is further influenced by the exact method and parameters of deposition[1]. Even for two nominally identical machines performing electron beam (e-beam) evaporation deposition, the parameters of deposition (surface properties, evaporation voltage and deposition rate, environment gasses) are generally not perfectly identical and will influence the final outcome of the index dispersion of the film. In turn, these small differences in refractive index can have a large impact on the optical properties of the resulting coating, especially within the domain of high precision optics. Therefore, for most technical applications of thin films, index determination is a topic of prime importance.

\footnotetext{
${ }^{*}$ Correspondence: riley.shurvinton@univ-amu.fr

Aix Marseille Univ, CNRS, Centrale Marseille, Institut Fresnel, Marseille, France

Full list of author information is available at the end of the article
}

The problem of index determination of metallic thin films is an especially challenging one, due to the presence of absorption, the significant and complex index dispersion relations, and potential inhomogeneities from metallic/dielectric mixing and oxide formation. In addition, previous work on metallic layer characterisation shows index dispersion tends to vary significantly with layer thickness $[2,3,4]$. Furthermore, different approaches are required depending on whether the metallic film in question is opaque or sufficiently thin to be semitransparent. Therefore, as of today, there is no single reliable and universal method to characterise these films. There is particular interest in a reliable spectrophotometric method, as these measurements do not require any specialised equipment, allowing simple direct determination of refractive index.

We will focus on the case of semitransparent absorbing thin-films, particularly within the visible range $(370-835 \mathrm{~nm})$. When the absorbing layer of interest is semitransparent rather than opaque, the layers below it will also contribute to the reflectance and transmittance spectra. Motivated by this, in 1999 Forouhi et al patented a novel approach for spectrophotometric characterisation of such layers that makes use of a multilayer structure[5]. The method was initially de- 
veloped for a DLC-like film, but it showed promise for characterisation of other absorbing semitransparent films. Instead of the traditional method of direct deposition of the metallic layer on a transparent substrate, the method utilises an opaque substrate coated with a dielectric layer of several hundred nanometers thickness, where both of these materials are wellcharacterised; and the semitransparent metallic layer is deposited on top (see fig. 1 for a schematic). The dielectric layer creates oscillations in the reflectance spectrum of the stack, which are then strongly modulated by the metallic toplayer, with a high degree of sensitivity to the precise index of this layer. It is thought that this extra information could improve the precision and accuracy of the fit. Indeed, a similar method making use of interference enhancement is known for ellipsometric methods of index determination $[6,7]$. Additionally, the use of an oscillatory structure has previously been demonstrated in some spectrophotometric techniques to enhance the available information in the problem of index determination $[8,9]$.

However, although this method was first introduced in 1999, it has not yet begun to see wide adoption in the thin-film community. One reason is that use of an opaque substrate restricts spectroscopic measurements to only reflectance, rather than both reflectance and transmittance as is possible with a transparent substrate. As the problem of index fitting is underdetermined[10], it is often recommended to use multiple different measurements during the fitting (for example, in both reflectance and transmittance, or at multiple angles of incidence or with different polarisation states of light) to minimise errors and improve accuracy[11]; so it was unknown whether the interference enhancement would provide a net positive to the overall accuracy of the fitting. The introduction of an additional thin film layer may also introduce uncertainties if this layer is not properly characterised. The patent provides a schematic overview of the method, but does not detail parameters such as the substrate choice and optimal dielectric thickness; in other words, it does not present a clear example of realisation of the method.

We present here a comparison and validation of this method for index dispersion determination when compared to the traditional method of deposition of the absorbing layer on a transparent substrate, and an assessment of the optimal parameters and tolerances of the method. We first use simulations to investigate the effect of approach and stack design on the accuracy and repeatability of the index determination. We then demonstrate an experimental verification of the method for semitransparent titanium thin films, using two models to approximate the refractive index dispersion of these films.

\section{Simulation Work: Comparison of two stack types for numerical index determination Method}

We began by using simulations to compare the performance of two stack designs. One employed this novel interference enhancement method, using an opaque substrate overcoated with a dielectric layer of several hundred nanometers thickness and a metallic layer with thickness chosen to be semitransparent (usually tens of nanometers, depending on the material). This design will here be referred to as the opaque substrate (OS) stack. Its performance is compared to the standard method for metallic thin-film index determination of deposition of the metallic layer directly on a transparent substrate, which will be referred to as the transparent substrate (TS) stack. Schematics of both stack designs and the resulting spectral features can be seen in fig. 1 and 2. The layers are modelled using nondispersive refractive indices chosen to be close to those of real materials. The index for the metallic top layer in both stacks is $2.54-3.43 i$, chosen to be similar to titanium at a representative wavelength of $549 \mu \mathrm{m}[12]$. A corresponding initial thickness of $40 \mathrm{~nm}$ was used for this layer. In the OS case, the index for the dielectric spacer layer was chosen to be 1.47, similar to the dielectric silicon dioxide, with a thickness of $800 \mathrm{~nm}$. The substrate index was $4.087-0.04 i$, similar to bulk silicon. In the TS case, the substrate index was 1.46 , similar to bulk silica glass.

We sought to study the effect of random multiplicative and additive noise on the spectra used for fitting, to mimic measurement errors and biases respectively in a real measurement. Using the refractive indices and thicknesses above, we generated a reflectance spectrum in the OS case, or a pair of reflectance and transmittance spectra in the TS case, where reflectance $\mathrm{R}$ and transmittance $\mathrm{T}$ take values between 0 and 1 . An amount of additive and multiplicative noise is applied to these spectra, and the simulation script then attempts to fit against these spectra to determine the refractive index of the metallic toplayer. In the TS case, both the transmittance and reflectance spectra are considered simultaneously, whereas the OS case is limited to reflectance only. If this limitation is significant and leads to the problem being underdetermined, it will therefore be very susceptible to the effects of noise and other random errors. Conversely, if the OS stack design provides more information as theorised due to higher sensitivity to the precise refractive index of the metallic layer, it should display more resistance to these errors compared to the TS stack. Therefore, 
assessing how this noise impacts the fit would allow us to judge the suitability of the method.

The stack designs (OS and TS) were simulated in MatLab using functions from the Thin-Film toolbox, developed by Ulf Griesmann [13]. The stacks are stored in a structure array, with each element of the structure representing a layer of the stack containing information about the layer thickness and refractive index. The toolbox uses the matrix transfer method to calculate reflectance and transmittance at each layer interface, over the chosen spectral range with a wavelength spacing of $5 \mathrm{~nm}$. For this initial analysis, we treated all of our materials as nondispersive, with the assumption that findings could be generalised to the case of dispersive materials. The question of index dispersion is discussed further in the next section.

The noise was generated using a normal distribution via MatLab's normrnd function[14]. For each spectrum, additive noise from a normal distribution with mean 0 and standard deviation 0.001 was added to each wavelength point to represent measurement bias. The spectrum was then multiplied by a bias value generated from a normal distribution with mean 1 and standard deviation 0.001 , to represent measurement error and noise. To ensure the sampled population of noise was itself approximately normally distributed, it was necessary to have a sufficiently large number of noisy spectra for each test[15]. Therefore this process was repeated 100 times for each configuration studied, generating 100 reflectance spectra for each OS case or 100 reflectance and 100 transmittance spectra in each TS case.

Index fitting for each noisy spectrum (or pair of spectra in the TS case) was performed via direct numerical optimization, using MatLab's nonlinear optimization function[16] with $n, k$ and the absorbing layer thickness $d$ as the parameters of optimization. The merit function to be minimized is $\frac{1}{N} \sqrt{\Sigma \delta^{2}}$ : the least-squares sum of the difference $\delta$ over $\mathrm{N}$ wavelength points between the target spectrum or spectra, and the trial spectrum or spectra generated at each step using trial values of $n, k$ and $d$. Fig. 3 illustrates the stages of the fitting procedure, showing the initial spectrum; the result of superimposed noise; and the resulting fit against the noisy data. The difference between the initial spectrum and the fit reflects a deviation in $n, k$ and $d$ between the nominal values used to generate the initial spectrum, and the optimized values found in the fit, due to the addition of noise to the spectrum.

This process is repeated 100 times for each configuration tested to generate a population of $n, k$ and $d$ triplets. Fig. 4 shows sample scatter plots for $100 n, k$ and $d$ values, generated using the OS case.

Since each sample population of 100 noisy spectra can be assumed to be equally normally distributed, the resulting variation in the populations of $n, k$ and $d$ will reflect the susceptibility of the fitting method to the noise. Greater variation suggests a higher susceptibility to noise, whereas smaller variation suggests the model is more robust. This effect can be assessed by calculating the standard deviation of the $n, k$ and $d$ values. The standard deviation can then be used as a figure of merit to assess the performance of the fitting model and the impact of stack design choices, such as thickness of different layers.

The uniqueness range of both methods was also calculated. This is a qualitative way to assess the uniqueness of a given optimum, as an underdetermined optimization problem may present multiple optimal solutions, but only one of them will be the desired 'true' solution. The uniqueness test varies one fit parameter within a range around its 'true' value, and optimizes the remaining free parameters to minimise the merit function. If the lowest merit function can only be obtained in a narrow window for the fixed parameter, then the solution is strongly unique. Conversely, if multiple widely-spread values for the fixed parameter yield the same merit function, then the solution is not unique and indeed the problem is poorly posed for this optimization method. As the index $(n-\mathrm{i} k)$ of a film may be correlated with its thickness $d, d$ was chosen as the fixed parameter in this analysis.

\section{Results}

The OS and TS stacks were both tested for noise susceptibility. Four configurations were trialed, for a metallic layer thickness of $40 \mathrm{~nm}$ : the OS stack in reflectance with $800 \mathrm{~nm}$ dielectric, calculated at normal incidence $\left(8^{\circ}\right)$; the TS stack in reflectance and transmittance, calculated at normal incidence; and both configurations with additional calculations at oblique incidence $\left(45^{\circ}\right)$ taken into account. The results are seen in Table 1.

Table 1 Standard deviation for 100 simulated cases of $n, k$ (both unitless) and $d(\mathrm{~nm})$ when using four different methods.

\begin{tabular}{cccc}
\hline Configuration & \multicolumn{3}{c}{ Standard Deviation } \\
& $\mathbf{n}$ & $\mathbf{k}$ & $\mathbf{d}$ \\
\hline OS normal incidence & 0.015 & 0.0116 & 0.236 \\
OS normal + oblique incidence & 0.0095 & 0.0064 & 0.132 \\
TS normal incidence & 0.5365 & 0.0207 & 5.5 \\
TS normal + oblique incidence & 0.0212 & 0.0081 & 0.223 \\
\hline
\end{tabular}

These results show that the improvement factor gained with the addition of oblique measurements depends on the stack (OS or TS). For the TS, the improvement in standard deviation when oblique measurements are taken into account is huge: a reduction of more than 20-fold in the standard deviation of $n$ 
and $d$, and a reduction by more than half in the standard deviation of $k$. This suggests that, without the oblique measurements, the TS method is somewhat reliable in determination of $k$ but extremely vulnerable to errors and bias in the determination of $n$ and $d$. Conversely, while an improvement is gained in the OS case by adding oblique measurements, it is more subtle - a reduction by less than half in standard deviation for all three of $n, k$ and $d$. Indeed, the performance of the OS method when only using normal incidence measurements is roughly equivalent to the performance to the performance of TS using both normal and oblique incidence measurements, with slightly less sensitivity to $k$ but better accuracy when determining $n$. As measurements at larger incidence can often be less reliable, due to factors such as larger beam area at glancing angles and potential fluctuations in polarisation state of incoming light, the comparative high reliability of the OS stack even when used only at normal incidence is a strong point in its favour.

Additionally, observing the scatter of $n, k$ and $d$ gives some insight to how these parameters behave during optimization, as seen in fig. 4. For instance, the plot of $n$ against $d$ shows a strong negative correlation. This suggests that the fitting method here is mostly sensitive to the product $n d$, and that an overestimate in $n$ may be compensated for by an underestimate in $d$ and vice-versa. A similar correlation is seen in $k$ against $d$, although the relation is less strong, suggesting that $k$ has a stronger uniqueness in the fitting method. $n$ and $k$ show an overall positive correlation, since an overestimate in $d$ is therefore likely to lead to an underestimate in both $n$ and $k$.

\section{Uniqueness range}

The uniqueness range of both the TS and OS stacks were calculated. A reference spectrum (or spectra in the TS case) was generated using $n=2.54, k=3.43$ and $d=40 \mathrm{~nm}$. The metallic layer thickness $d$ was chosen as the fixed parameter, which was varied around its optimal value of $40 \mathrm{~nm}$. The other parameters $n$ and $k$ were then optimized against the reference spectrum or spectra.

The evolution of the least-squares merit function, given by $\frac{1}{N} \sqrt{\Sigma \delta^{2}}$ (for $\delta$ the difference between the optimized and reference spectra at each point for $\mathrm{N}$ points), as $d$ is varied between 35 and $45 \mathrm{~nm}$ for the OS and TS cases can be seen in fig. 5. For the OS case, the merit rises sharply as $d$ moves away from the optimum of $40 \mathrm{~nm}$, suggesting a strong uniqueness. Conversely, for the TS case the merit increases slowly as $d$ is varied from the optimum, suggesting a weaker uniqueness and a lower sensitivity to the parameters of the fit. This supports our prediction that the OS stack design is more likely to result in convergence to a single unique solution.

These promising initial results indicated that there was merit to the OS approach for index determination. Therefore, we chose to move forward with the case of OS at normal incidence in our simulations. We chose not to use oblique measurements, as the results in Table 1 suggested the method was stable without them, and in experiments they may contribute to an additional source of error. We then investigated the impact of certain stack design choices within this approach, such as thicknesses of the various layers and effect of material choices.

\section{Impact of metallic layer thickness}

We assessed the range of Ti thicknesses over which this method can be implemented, and the effect this has on the fit. For our chosen method of thin film deposition (electron beam evaporation), the lower thickness limit is in practical terms between 5 and $10 \mathrm{~nm}$, and we cannot reliably deposit layers of lower thickness which are homogeneous, uniform and continuous. Conversely, for sufficiently thick absorbing layers, the spectral oscillations of the dielectric spacer layer are strongly washed out, and the spectrum tends towards that of the TS stack with the same metallic thickness - ie, the contributions of the substrate are minimised. The thickness at which this occurs varies depending on the material in question; based on simulations, we found it to be around $100 \mathrm{~nm}$ for titanium with the assumption $\mathrm{k} \sim 3$. We therefore analysed thicknesses between 10 and $100 \mathrm{~nm}$ for the simulated Ti layer.

The evolution in the standard deviation as the layer thickness changes can be seen in fig. 6 . The optimal thickness for the OS stack is shown to be $30-40 \mathrm{~nm}$. In particular, for metallic layer thicknesses above $\sim 60 \mathrm{~nm}$ the standard deviation increases sharply, suggesting that as the spectral oscillations become weaker, the sensitivity to the precise index of the absorbing layer becomes lower. Equally, for layer thicknesses below $20 \mathrm{~nm}$ the standard deviation also increases. This may be due to the fact that these thinner layers have a much smaller impact on the overall reflectance spectrum, and these features can more easily be obscured by noise. This emphasizes the importance of minimising errors for characterisation of very thin layers.

\section{Impact of dielectric layer}

The optimum index and thickness of the dielectric layer was also an important question. Oscillations in the reflectance spectrum of the OS occur due to interference within the dielectric layer. By analogy with a Fabry-Perot etalon, troughs in $\mathrm{R}$ occur when the 
phase difference per round trip, $\phi=2 n d$ where $\mathrm{d}$ is the thickness of the dielectric layer and $n$ is the real part of its refractive index, is an integer multiple of the wavelength $\lambda$; and peaks occur when $\phi$ is instead an odd multiple of $\frac{\lambda}{2}$. Therefore, the number of peaks visible within a given wavelength range depends on the thickness $d$ and refractive index $n$ of the dielectric layer, which may impact the accuracy and sensitivity of the fitting.

Thicknesses of the dielectric layer between 100 and $1500 \mathrm{~nm}$ were trialled for the OS stack, corresponding to an optical thickness range of 146-2190 nm for the silica-like dielectric layer. The standard deviation in the determined $\mathrm{n}$ and $\mathrm{k}$ of the metallic layer was again used for the assessment of merit. Standard deviation was seen to fluctuate significantly with the optical thickness, with stronger fluctuation at lower optical thicknesses, and smaller fluctuations for larger thicknesses (optical thickness > $1000 \mathrm{~nm}$, corresponding to physical thickness $>700 \mathrm{~nm}$ ). Similar trends were seen when an index of 2.3 was instead used for the dielectric layer, representing the real dielectric niobia $\left(\mathrm{Nb}_{2} \mathrm{O}_{5}\right)$. This suggests that different dielectrics will give approximately equivalent behaviour, and the main contribution of the dielectric layer is its optical thickness. We concluded that dielectric thickness and index both did not have a large impact on the fitting, as long as the optical thickness was sufficient to show at least one full oscillation. This was investigated further with our physical samples.

Optimal wavelength spacing was similarly chosen by evaluating the resulting change in the standard deviation. It was found that $5 \mathrm{~nm}$ wavelength spacing was sufficient to minimise the standard deviation without significantly slowing computation time.

These results suggested the OS fitting regime provided a viable alternative to the transparent stack, and indeed could even offer an improvement based on the simulations. We then proceeded with experimental verification using for $\mathrm{Ti}$ thin films.

\section{Experimental work: Index determination of thin Ti layers using interference enhancement}

The simulation work detailed above only gave part of the picture regarding the performance of the OS stack design. Crucially, it did not consider the dispersion of the materials in question. Real materials, especially metals, have refractive indices that can vary strongly with wavelength, and this makes the problem of index fitting considerably more complicated. It was therefore important to verify these results using real materials.

\section{Methods}

Sample deposition and measurement

We chose titanium (Ti) as our metal whose index would be investigated. Commercial 4" silicon ( $\mathrm{Si}$ ) wafers were used as the opaque substrate, as they were stable and had well-known and repeatable dispersion properties. The wafers were cut into pieces approximately $1 \times 2$ " in size to form the samples. For the dielectric layer we used silica $\left(\mathrm{SiO}_{2}\right)$, as it is well-calibrated and repeatable and had natural compatibility with the substrate material, since Si naturally forms a thin layer of oxide when exposed to air.

Samples were deposited in a Bühler-Leybold Optics SYRUSpro 710 deposition machine, which uses electron beam evaporation in a vacuum of $2 \times 10^{-7}$ mbars. The deposition rates were $0.5 \mathrm{~nm} / \mathrm{s}$ for $\mathrm{SiO}_{2}$, and $0.05 \mathrm{~nm} / \mathrm{s}$ for $\mathrm{Ti}$. The slow deposition rate allowed for high accuracy and repeatability of the deposited Ti thickness. The layer thickness was monitored using a quartz microbalance.

The reflectance spectra of the samples were measured using a Perkin-Elmer Lambda 1050 spectrophotometer. The wavelength range was $370-835 \mathrm{~nm}$, and the wavelength spacing was $5 \mathrm{~nm}$. Measurements were taken at as close to normal incidence as possible, approximately $8^{\circ}$.

Description of index dispersion with a model

The indices of real materials are typically dispersive. Therefore the refractive index must be determined not just as a single value, but for a sufficiently large number of wavelength points depending on the shape of the dispersion and the wavelength range of interest. However, determining the index dispersion separately at hundreds of points is computationally expensive and often prone to errors, leading to unphysical or strangely shaped distributions.

An alternative approach is to instead proceed using a function, or model, which describes the index dispersion using several parameters. The parameters of the model then become the parameters of optimization, reducing the number of values to be optimized from tens or hundreds to just a few. For this reason, use of a dispersion model is often highly advantageous. However, care must be taken to choose an appropriate model to describe the material in question, especially as metals often have complex dispersion relationships. The models used in this work to describe the semitransparent Ti layer are described below.

\section{Refractive index models for thin Ti layer \\ 4-parameter analytical model}

Initial investigation of the Ti layer was made using a simple 4-parameter analytical model to approximate 
the index dispersion, obtained by testing simple geometric functions derived from observing the shape of the index dispersion of bulk titanium. From the functions we trialled, it was found that the most suitable fit was given using a linear function of wavelength for $n$ and a reciprocal function of wavelength for $k$ :

$$
n=A+B \lambda \quad k=C+\frac{D}{\lambda}
$$

where $A, B, C$ and $D$ are the analytical parameters to be determined.

\section{Combined Drude and Forouhi-Bloomer model}

The Ti layer was also described using the modified Drude model, which describes $n$ and $k$ of a metal using three parameters: $E_{\gamma 0}, E_{D}$ and $s$ (see [17], section VIII A for a full description). However, the Drude model on its own was found to be insufficient to describe the index of the layer. As such, we further extended it by combination with dielectric models[18]. The best performance was found using a combination of the modified Drude model with the Forouhi-Bloomer model.

The Forouhi-Bloomer model [19] is a physical model derived from interband transitions within a solid. The model uses one or multiple absorbent transitions to determine $\mathrm{k}$ :

$k(E)= \begin{cases}\left(A\left[E-E_{g}\right]^{2}\right) /\left(E^{2}-B * E+C\right) & E>E_{g} \\ 0 & E \leq E_{g}\end{cases}$

In testing, it was found that describing a single transition within our wavelength range was sufficient. The real part can then be obtained via the Kramers-Kronig relations[20] by adding one extra parameter $n_{\text {inf }}$ (see [19] for the full derivation). This gives $A, B, C, E_{g}$ and $n_{\text {inf }}$ as the five Forouhi-Bloomer parameters to be optimised in this model. $E_{g}$ is the band gap energy of the absorber and $n_{i n f}$ is the high-energy refractive index of the material (one or larger). Initial guesses for the other parameters can be made by observing their physical origin[21]:

- $A$ (in $e V)$ is derived from the square of the dipole matrix, and it gives the strength of the absorption peak. $A$ and $C$ are correlated, describing respectively the height and the width of the peak. As such, the larger $A$ is, the smaller $C$ becomes. Generally, $0<A<24$.

- $B / 2$ (in $\mathrm{eV}$ ) is approximately the center of the absorption peak. Generally, $3<B<30$.
- $C$ (in $e V^{2}$ ) is related to the width of the absorption peak. Generally, $3<C<150$.

The contributions of the Drude and Forouhi-Bloomer models can be combined by summation. Hence, $\epsilon_{\text {total }}=$ $\epsilon_{M D M}+\epsilon_{F B}$, where permittivity and refractive index are related by $\epsilon=(n+i k)^{2}$.

Results

Several stack designs consisting of a thick layer of silica $\left(\mathrm{SiO}_{2}\right)$ and a thin layer of titanium $(\mathrm{Ti})$ were deposited on silicon wafers using electron beam evaporation. Based on the simulation work, three thicknesses of $\mathrm{SiO}_{2}$ were initially trialed: $300 \mathrm{~nm}, 500 \mathrm{~nm}$ and $800 \mathrm{~nm}$. The titanium layer deposited on these samples was expected to be $28 \mathrm{~nm}$ thick based on initial calibration of our quartz microbalance.

The index fitting proceeds similarly to the theoretical case, using the measured spectra of the samples as the target and with the refractive index dispersion model parameters as the parameters of optimization, and seeking to minimise the least-squares merit function $\frac{1}{N} \sqrt{\Sigma \delta^{2}}$.

\section{Confirmation of $\mathrm{Si}$ and $\mathrm{SiO}_{2}$ index}

We chose the materials of commercial Si wafers for the substrate and $\mathrm{SiO}_{2}$ for the dielectric spacer layer due to them being reliable and repeatable, but it was still necessary to first carefully characterise these materials, as any inaccuracies in the values of their index dispersions would propagate through to the final estimate for the refractive index.

For the Si substrate, we used literature values for the refractive index dispersion. The database used was from the Sopra (SA) company in France [22]. The reflectance spectrum generated using these data was very close to the measured reflectance spectrum of the wafers, showing an average divergence of approx. $0.1 \%$ and a total least-squares merit of $0.897 \times 10^{-4}$. Hence, these data were deemed sufficient to use in our model.

However, the approach of using literature values was not suitable for the $\mathrm{SiO}_{2}$ layer. Thin-film properties typically differ from those of the bulk material, and further vary depending on the precise parameters of deposition. Additionally, the thickness of the deposited layer may vary with a tolerance of $\sim 1$ percent depending on the sample's position in the machine. Since the OS fitting method is very sensitive to the optical thickness of the dielectric layer, it was necessary to individually determine the parameters of the $\mathrm{SiO}_{2}$ layer for each sample.

In order to allow separate measurement and characterisation of both the $\mathrm{SiO}_{2}$ and the Ti layers, the Ti layer was only deposited on part of each sample. This was achieved by partial masking in between deposition of the $\mathrm{SiO}_{2}$ layer and the Ti layer. Samples 
were cut along the tangent to the direction of rotation within the deposition machine to take advantage of the higher uniformity in this direction. This ensured that the variation in $\mathrm{SiO}_{2}$ layer thickness between the two parts of the sample was minimised to less than $0.1 \%$.

For the index of the $\mathrm{SiO}_{2}$ layer, a two-parameter Cauchy model $[23,24]$ was used for the real part of the refractive index, with the imaginary part taken to be 0 (valid for silica in the visible range [23]). The thickness of the layer is also a free parameter in this fitting. The Cauchy approximation for the $\mathrm{SiO}_{2}$ layer was optimized using nonlinear function minimization, and the resulting calculated spectra is shown in fig. 7 alongside the measured spectra used for the fitting. Although simple, the Cauchy approximation shows good agreement against the measured data, with maximum divergence 1-2\% and a typical least-squares merit of $3.90 \times 10^{-4}$. This fitting was performed for every sample to precisely determine the thickness before fitting for the $\mathrm{Ti}$ index.

\section{Index determination of metallic layer}

Several models were tested for the approximation of the index of the Ti layer, including several analytical models using geometric functions, and physical models such as the Drude model and the combined DrudeLorentz model. We chose to closely investigate two: the four-parameter geometric model detailed in equation (1), and a combined Modified Drude and ForouhiBloomer (MD-FB) model. The resulting fits from these models for a Ti layer of nominal $28 \mathrm{~nm}$ thickness can be seen in fig. 8. The thickness obtained from fitting via each model was $27.2 \mathrm{~nm}$ for the four-parameter model, and $26.9 \mathrm{~nm}$ for the FB-MD model.

Reduction of the parameter space reduces the degrees of freedom of the fit and improves the likelihood of converging to a unique solution. Therefore, the fourparameter geometric model was initially used to fit the Ti layer in our investigation. The least-squares merit function achieved with this model is $3.66 \times 10^{-4}$, indicating a very good fit to the experimental data. However, if one looks closer there are several places, for example the troughs at 500-540 nm and 620-700 nm, where the fitting clearly diverges from the measured spectrum, motivating the use of a more complex model to investigate whether improvements could be made.

The only model that was found to give comparable performance to the four-parameter model for the index of the $\mathrm{Ti}$ layer was the combination of the Modified Drude and Forouhi-Bloomer models (MDFB). This model gave a slightly improved least-squares merit function for these data of $3.20 \times 10^{-4}$. Comparison with the four-parameter model fit in fig. 8 shows that it gives closer agreement with the measured data at shorter wavelengths, but diverges further from the data at longer wavelengths (namely at the trough between 415-440 nm).

The results given by both models for the index dispersion of the $28 \mathrm{~nm}$ Ti layer are seen in the bottom graph of fig. 8, along with the literature data for bulk Ti for comparison. Both the simple model and the MD-FB model give similar approximations for the index dispersion of the $\mathrm{Ti}$ layer. These results generally agree with other work on characterisation of $\mathrm{Ti}$ thin films [25]. Furthermore, as was previously demonstrated, the OS stack design exhibits a high uniqueness in its fitting.

However, despite the improvements achieved with the MD-FB model, the fitting still shows some divergence at the peaks and troughs of the spectrum. It is possible that this is due to errors introduced from the refractive index values used for the materials of silicon and silica. Indeed, the merit function obtained for the $\mathrm{SiO}_{2}$ layer fitting is larger than that obtained for the Ti layer $\left(3.90 \times 10^{-4}\right.$ vs $\left.3.20 \times 10^{-4}\right)$. As such, the $\mathrm{Ti}$ fit returning a lower merit function than this may not actually be an indication of a better fit, but rather a compensation by the model for errors. In this way, paradoxically, a lower merit function may indicate an unphysical fit. This reflects one of the problems with using models with a larger parameter space, as it enlarges the degeneracy of the solution space and increases the possibility of fits with equal or improved merit functions being inaccurate or unphysical. However, close agreement with the results of the simpler 4-parameter model, and strong repeatability, indicate that the MD-FB model is performing well.

We can use the repeatability of the fitting and the previous estimates of uniqueness to estimate the tolerances of this method. From the calculations of the uniqueness range, a least-squares merit of $\sim 3 \times 10^{-4}$ corresponds to a range in thicknesses of around $\pm 10 \%$. If we expect this to correspond to a similar range in $\mathrm{n}$ and $\mathrm{k}$, then this suggests we can expect around $10 \%$ variation in $\mathrm{n}$ and $\mathrm{k}$ as well, or a variation of around \pm 0.2 . From experiments, when multiple samples were analysed, deposited in the same conditions with the same $28 \mathrm{~nm}$ thick Ti layer, we found the method was repeatable to around \pm 0.1 in $n$ and $k$ and $\pm 1 \mathrm{~nm}$ in layer thickness $d$, corresponding to around $\pm 5 \%$ for all three parameters.

The effect of the $\mathrm{SiO}_{2}$ layer thickness on the performance of the OS stack was also tested experimentally. Three Si samples with different thicknesses of $\mathrm{SiO}_{2}$ (300, 500 and $800 \mathrm{~nm}$ ) were all coated simultaneously with a Ti layer of $28 \mathrm{~nm}$ nominal thickness. Fitting was performed on these samples to determine $\mathrm{n}$ and $\mathrm{k}$. 
We report in Table 2 the parameters for the index dispersion of Ti for these samples using the simple fourparameter model. It is seen that parameters $\mathrm{C}$ and $\mathrm{D}$ are very close to within \pm 0.1 and \pm 0.03 , respectively. Parameters $\mathrm{A}$ and $\mathrm{B}$ show more variation, likely because within this wavelength range they can strongly compensate for one another. The resulting approximations for $\mathrm{n}$ and $\mathrm{k}$ were within \pm 0.1 , in agreement for our previous determination of the tolerance, and the estimations for the Ti layer thickness were within $\pm 1 \mathrm{~nm}$. Equally, the MD-FB model was found to give a very similar result for index dispersion for each sample, determining index to within \pm 0.13 for all values of $\mathrm{n}$ and $\mathrm{k}$ and layer thickness $\mathrm{d}$ to within $\pm 0.7 \mathrm{~nm}$. This result shows stability of the technique, and corroborates the prediction from simulations that above $\sim 250 \mathrm{~nm}$ the dielectric thickness does not have a large impact on the fitting. We concluded that for a given thickness of metallic Ti layer, this method showed good repeatability.

Table 2 Parameters A, B, C and D and layer thickness $d$ for fitting Ti layer using the four-parameter model (equation 1) for three different $\mathrm{SiO}_{2}$ thicknesses $(300,500$ and $800 \mathrm{~nm})$.

\begin{tabular}{lccccc}
\hline $\begin{array}{l}\mathrm{SiO}_{2} \\
\text { thickness }\end{array}$ & A & B $\left(\mu \mathbf{m}^{-1}\right)$ & C & D $(\mu \mathbf{m})$ & d (nm) \\
\hline 300 & 0.546 & 3.52 & 4.05 & -0.654 & 28.5 \\
500 & 0.897 & 2.99 & 3.98 & -0.685 & 28.9 \\
800 & 0.779 & 3.41 & 4.13 & -0.650 & 27.2 \\
\hline
\end{tabular}

Of note, the values for the index dispersion of the Ti thin-film layer are significantly different from the literature values for bulk Ti. The real part $\mathrm{n}$ is slightly higher, and the imaginary part $\mathrm{k}$ is much lower. It is not uncommon for the index of thin film materials to differ substantially from that of the bulk material, due to a variety of phenomena. One possible effect that is likely to have a large impact on Ti thin film layers is the formation of oxides at the upper air/layer boundary.

If these oxide layers are indeed present and have some effect on the refractive index of the layer, it is also likely that their impact would vary with the thickness of the deposited layer, since if oxidation depth is independent of layer thickness, the proportion of oxide formation varies with the total layer thickness. In addition, several other metals (such as gold [3]) are known to exhibit additional index variation with thickness independent of oxide formation, which arise from various factors such as crystalline grain formation. This motivated investigation of the index of Ti films of different thicknesses.

\section{Impact of Ti layer thickness}

The OS fitting method was used to investigate the change in refractive index of titanium with layer thickness. We deposited samples using $800 \mathrm{~nm} \mathrm{SiO}_{2}$ and a range of 6-70 nm Ti thicknesses. The index was fit using the combined MD-FB model.

The results of this fitting are shown in fig. 9, which displays the evolution with layer thickness of the refractive index at a representative wavelength of $450 \mathrm{~nm}$. As the index dispersion varies gradually over the wavelength range, this is sufficient to describe the trend. For layers between 17-40 nm, an increase in layer thickness corresponds with an increase in $n$ and $k$, from $\sim 2$ to $\sim 2.3$ and from $\sim 2$ to $\sim 2.5$ at $450 \mathrm{~nm}$, respectively.

Within this range there are noticeable fluctuations of around \pm 0.2 from this trend, which are slightly larger than the repeatability tolerances of \pm 0.1 previously found. This may suggest slight instability in the layer index due to potentially slightly different deposition conditions between samples.

The thinnest layer investigated $(\sim 6 \mathrm{~nm})$ and the thickest $(\sim 70 \mathrm{~nm})$ both show significantly different behaviour. The $6 \mathrm{~nm}$ layer exhibits a much lower absorbance than the thicker layers. This is possibly due to oxidation of a comparatively large proportion of the layer, forming the nonabsorbing dielectric $\mathrm{TiO}_{2}$. This corresponds with other work on Ti thin films suggesting that the oxide thickness formed in air at room temperature is on the order of 3-6 $\mathrm{nm}[26]$. As $\mathrm{Ti}$ and $\mathrm{TiO}_{2}$ have significantly different index dispersions, presence of a large proportion of both within the layer suggests substantial inhomogeneities within the layer. As such, the models described above, which treat all layers as homogeneous with regards to index dispersion, may not be sufficient to fully describe this layer. It is possible that a more detailed assessment of this layer could be achieved using a model that considers these inhomogeneities. However, such analysis is outside of the scope of this paper, and we instead conclude that the obtained result represents the effective index of this layer if treated as homogeneous.

In contrast to the layers of $17-40 \mathrm{~nm}$ thickness, the $70 \mathrm{~nm}$ layer does not follow the trend of increasing index with thickness. This suggests that for thicker layers, a simple linear trend is no longer sufficient to describe the evolution in index dispersion with layer thickness. However, as this layer is close to the absorbing limit for the OS method, investigation of thicker Ti layers $(100 \mathrm{~nm}+)$ is not compatible with the proposed technique.

\section{Limitations of the fitting method}

The effective lower limit for the layer thickness in this work was the thinnest layer we could reliably deposit using our process (electron beam deposition in Bühler Leybold Optics SYRUSpro 710 deposition machine) while still ensuring a uniform and homogeneous layer. This represents a thickness limit of 5-6 nm. 
The upper thickness limit for this method arises due to absorbance. From theoretical concerns, as the thickness of the top metallic layer increases, the oscillations of the stack below become strongly washed out, and this fitting procedure is therefore less suitable. As such, we restricted our investigation to layers of $70 \mathrm{~nm}$ thickness or less. Refinement of the model and method could potentially improve this. However, for many applications such as transmission filters [27, 28], antireflection coatings [29] and absorbers [30], and colour coatings [31,32], a semitransparent absorbing layer is ideal, and this method of index determination is wellsuited to characterise such layers.

\section{Conclusion}

A spectrophotometric method of determining refractive index for a semitransparent absorbing layer was introduced. This method uses a dielectric spacer layer between the opaque substrate and the absorbing toplayer to generate an oscillatory reflectance spectrum. These oscillations enhance the sensitivity of the spectrum to the refractive index dispersion of the absorbing layer, providing more information in the fitting. In simulations, the method was compared against the standard method of depositing the semitransparent layer on a transparent substrate using simulations. We found that the opaque substrate method is more robust and resistant to noise than the method of using a transparent substrate, and shows improved uniqueness, being more likely to converge to a single solution. An experimental verification of the method was carried out for the case of Ti thin films between 6-70 nm in the visible spectrum $(370-835 \mathrm{~nm})$. The Ti film was deposited on a silicon substrate over a layer of silicon dioxide, fabricated in-house using electron beam evaporation. In experimental testing, this method can quickly compute dispersive refractive index of a titanium sample with repeatability to within $\pm 5 \%$. The effect of layer thickness on the index of titanium thinfilms was found to be roughly linear, with the thinnest film studied $(6 \mathrm{~nm})$ showing a large divergence in refractive index.

\footnotetext{
Acknowledgements

The authors would like to thank Ulf Griesmann, the developer of the open-source Thin Film Toolbox. The scripts in this toolbox were used for much of this work. We also thank Abdul R Forouhi for contributions to the discussion and sharing insights on the index determination process.

Funding

This work was completed in the course of a $\mathrm{PhD}$ project funded by the Ecole Doctorale 352 of I'Ecole Centrale Marseille and Aix-Marseille University.

Abbreviations

OS (opaque stack); TS (transparent stack); MD-FB (modified Drude and Forouhi-Bloomer)
}

\section{Availability of data and materials}

The datasets used and/or analysed during the current study are available from the corresponding author on reasonable request.

Ethics approval and consent to participate

Not applicable

Competing interests

The authors declare that they have no competing interests.

Authors' contributions

R. Shurvinton wrote most of the manuscript. A. Moreau assisted with creation of samples. F. Lemarchand assisted with simulations and theoretical work. F. Lemarchand and J. Lumeau supervised the work. All authors were involved in editing, proofreading and discussion.

\section{Author details}

Aix Marseille Univ, CNRS, Centrale Marseille, Institut Fresnel, Marseille, France.

\section{References}

1. Dobrowolski, J.A., Ho, F.C., Waldorf, A.: Determination of optical constants of thin film coating materials based on inverse synthesis. Applied Optics 22(20), 3191-3200 (1983)

2. Cathelinaud, M., Lemarquis, F., Amra, C.: Index determination of opaque and semitransparent metallic films: application to light absorbers. APPLIED OPTICS 41(13), 2546-2554 (2002)

3. Yakubovsky, D.I., Arsenin, A.V., Stebunov, Y.V., Fedyanin, D.Y., Volkov, V.S.: Optical constants and structural properties of thin gold films. OPTICS EXPRESS 25(21), 25574-25587 (2017)

4. Solis-Tinoco, V., Acevedo-Barrera, A., Vazquez-Estrada, O., Munguia-Cervantes, J., Hernandez-Como, N., Olguin, L.F., Garcia-Valenzuela, A.: Fast and accurate optical determination of gold-nanofilms thickness. Optics and Laser Technology 134(106604), 985-989 (2021)

5. Li, G., Zhu, H., Harrison, D.A., Forouhi, A.R., Xu, W.: United States Patent no. US 6,392,756 B1; Method and Apparatus for Optically Determining Physical Parameters of Thin Films Deposited on a Complex Substrate (2002)

6. McGahan, W.A., Johs, B., Woollam, J.A.: Techniques for ellipsometric measurement of the thickness and optical constants of thin absorbing films. Thin Solid Films 234(1-2), 443-446 (1993)

7. Hilfiker, J.N., Singh, N., Tiwald, T., Convey, D., Smith, S.M., Baker, J.H., Tompkins, H.G.: Survey of methods to characterize thin absorbing films with spectroscopic ellipsometry. Thin Solid Films 516(22), 7979-7989 (2008)

8. Luňáček, J., Hlubina, P., Luňáčková, M.: Simple method for determination of the thickness of a nonabsorbing thin film using spectral reflectance measurement. APPLIED OPTICS 48(5) (2009)

9. Poelman, D., Smet, P.F.: Methods for the determination of the optical constants of thin films from single transmission measurements: a critical review. Journal of Physics D: Applied Physics 36, 1850-1857 (2003)

10. Ventura, S.D., Birgin, E.G., Martínez, J.M., Chambouleyron, I.: Optimization techniques for the estimation of the thickness and the optical parameters of thin films using reflectance data. Journal of Applied Physics 97, 043512-112 (2005)

11. Tikhonravov, A.V., Trubetskov, M.K., Sullivan, B.T., Dobrowolski, J.A.: Influence of small inhomogeneities on the spectral characteristics of single thin films. APPLIED OPTICS 36(28), 7188-7198 (1997)

12. Johnson, P.B., Christy, R.W.: Optical constants of transition metals: Ti, v, cr, mn, fe, co, ni, and pd. Phys Rev B 9(12), 5056 (1974)

13. Griesmann, U.: Thin Film Toolbox (2015). https://sites.google.com/site/ulfgri/numerical/thin-films Accessed Accessed 7th April 2020

14. Matlab Documentation - normrnd. mathworks.com/help/stats/normrnd.html Accessed 13th January 2021

15. Illowsky, B., Dean, S.: The Central Limit Theorem (2021). https://stats.libretexts.org/@go/page/696 Accessed Accessed 15th April 2021 
16. Matlab Documentation - fmincon https://www.mathworks.com/help/optim/ug/fmincon.html Accessed 10th January 2021

17. Hamberg, I., Granqvist, C.G.: Evaporated sn-doped in2o3 films: Basic optical properties and applications to energyefficient windows. Journal of Applied Physics 60, 123-160 (1986)

18. Lai, F., Lin, L., Gai, R., Lin, Y., Huang, Z.: Determination of optical constants and thicknesses of in2o3:sn films from transmittance data. Thin Solid Films 515(18), 7387-7392 (2007)

19. Forouhi, A.R., Bloomer, I.: Optical properties of crystalline semiconductors and dielectrics. Physical Review B 38(3), 1865-1874 (1988)

20. de L Kronig, R.: On the theory of dispersion of x-rays. Journal of the Optical Society of America 12(6), 547-557 (1926)

21. Yvon, J.: Forouhi-bloomer alias amorphous dispersion formula. Horiba Technical Notes 13 (2006)

22. Sopra S.A. http://www.sspectra.com/sopra.html Accessed 24th July 2015

23. Šimurka, L., Čtvrtlík, R., Tomaštík, J., Bektaș, G., Svoboda, J., Bange, K.: Mechanical and optical properties of sio2 thin films deposited on glass. Chemical Papers 72, 2143-2151 (2018)

24. Gooch, J.W.: Cauchy's dispersion formula. In: Encyclopedic Dictionary of Polymers. Springer, New York, NY (2007)

25. Belousov, D.A., Terent'ev, V.S., Spesivtsev, E.V., Korolkov, V.P.: Spectral data of refractive index and extinction coefficient for thin films of titanium group metals used for fabrication of optical microstructures. Data in Brief 28, 104903 (2020)

26. Matveev, V.A., Pleshanov, N.K., Bulkin, A.P., Syromyatnikov, V.G.: The study of the oxidation of thin ti films by neutron reflectometry. In: March (ed.) 5th European Conference on Neutron Scattering, vol. 340 (2011)

27. Dobrowolski, J.A., Li, L., Kemp, R.A.: Metal/dielectric transmission interference filters with low reflectance. 1. design. Applied Optics 34(25), 5673-5683 (1995)

28. Berning, P.H., Turner, A.F.: Induced transmission in absorbing films applied to band pass filter design. Journal of the Optical Society of America 47(3), 230-239 (1957)

29. Zheng, Y., Kikuchi, K., Yamasaki, M., Sonoi, K., Uehara, K.: Two-layer wideband antireflection coatings with an absorbing layer. Applied Optics 36(25), 6335-6338 (1997)

30. Lemarquis, F., Marchand, G.: Analytical achromatic design of metal-dielectric absorbers. Applied Optics 38(22), 4876-4884 (1999)

31. Amotchkina, T.V., Sancho-Parramon, J., Janicki, V., Trubetskov, M.K., Zorc, H., Tikhonravov, A.V.: Design of multilayer coatings containing metal island films. Advances in Optical Thin Films IV Proc. SPIE 8168(816809) (2011)

32. Talagrand, C., Triggs, G., Bandhu, L., Garcia-Castillo, S., Broughton, B., Bhaskaran, H., Hosseini, P.: Solid-state reflective displays ( $\operatorname{srd} \cap$ ) for video-rate, full color, outdoor readable displays. Journal of the Society for Information Display 26(10), 619-624 (2018)

Figures

\section{fig1}

Figure 1 Schematic for the OS (opaque substrate) stack design. Inset shows the typical shape of the resulting reflectance spectrum.

Figure 2 Schematic for the TS (transparent substrate) stack design. Insets show the typical shapes of the resulting reflectance and transmittance spectra.

\section{fig3}

Figure 3 The stages of fitting to determine noise resistance: the initial simulated reflectance spectrum (blue), here for the OS stack; a generated noisy spectrum (red); and the resulting fit (purple, dashed).

\section{$f 4 a f 4 b f 4 c$}

Figure 4 Three sample scatter plots of $100 n, k$ and $d$ values for the metallic toplayer from fitting against spectra with normally distributed additive $(\mu=0, \sigma=0.001)$ and multiplicative $(\mu=1, \sigma=0.001)$ noise, for the OS stack with a $800 \mathrm{~nm}$ dielectric layer and a $40 \mathrm{~nm}$ metallic toplayer. Top, $\mathrm{k}$ against $\mathrm{n}$; mid, $\mathrm{n}$ against $\mathrm{d}$; bottom, $\mathrm{k}$ against $\mathrm{d}$. The cross on each plot indicates the nominal values used to generate the initial spectrum of $n=2.54, k=3.43$ and $d=40 \mathrm{~nm}$. The standard deviation for these data is 0.015 in $n, 0.0116$ in $k$ and 0.236 , corresponding to the first row in Table 1.

\section{fig5}

Figure 5 Evolution of least squares merit function with $d$ fixed and varied around from the optimum at $40 \mathrm{~nm}$, for the two fitting cases (OS and TS).

\section{fig6}

Figure 6 Evolution of standard deviation of determined $\mathrm{n}$ (solid) and $\mathrm{k}$ (dashed) for different simulated thicknesses of the metallic toplayer.

\section{fig7}

Figure 7 Measured reflectance spectrum from Si substrate coated with $800 \mathrm{~nm} \mathrm{SiO} 2$ (dashed) at near-normal incidence, and the resulting fit for the index of the $\mathrm{SiO} 2$ layer using a two-parameter Cauchy approximation (solid).

\section{f8a f8b}

Figure 8 Top: measured reflectance spectrum for Si/800 nm $\mathrm{SiO} 2 / 28 \mathrm{~nm} \mathrm{Ti}$ stack at near-normal incidence, and resulting fits for index dispersion of the Ti layer using simple and MD-FB models. Bottom: comparison between literature data for bulk $\mathrm{Ti}$ (dashed), and simple and MD-FB models for index dispersion of $28 \mathrm{~nm} \mathrm{Ti}$ layer (solid).

\section{fig9}

Figure 9 Evolution of determined $\mathrm{n}$ and $\mathrm{k}$ for different thicknesses of a $\mathrm{Ti}$ layer, deposited on top of an Si substrate coated with $800 \mathrm{~nm} \mathrm{SiO} 2$, shown at a single representative wavelength of $450 \mathrm{~nm}$. 
Figures

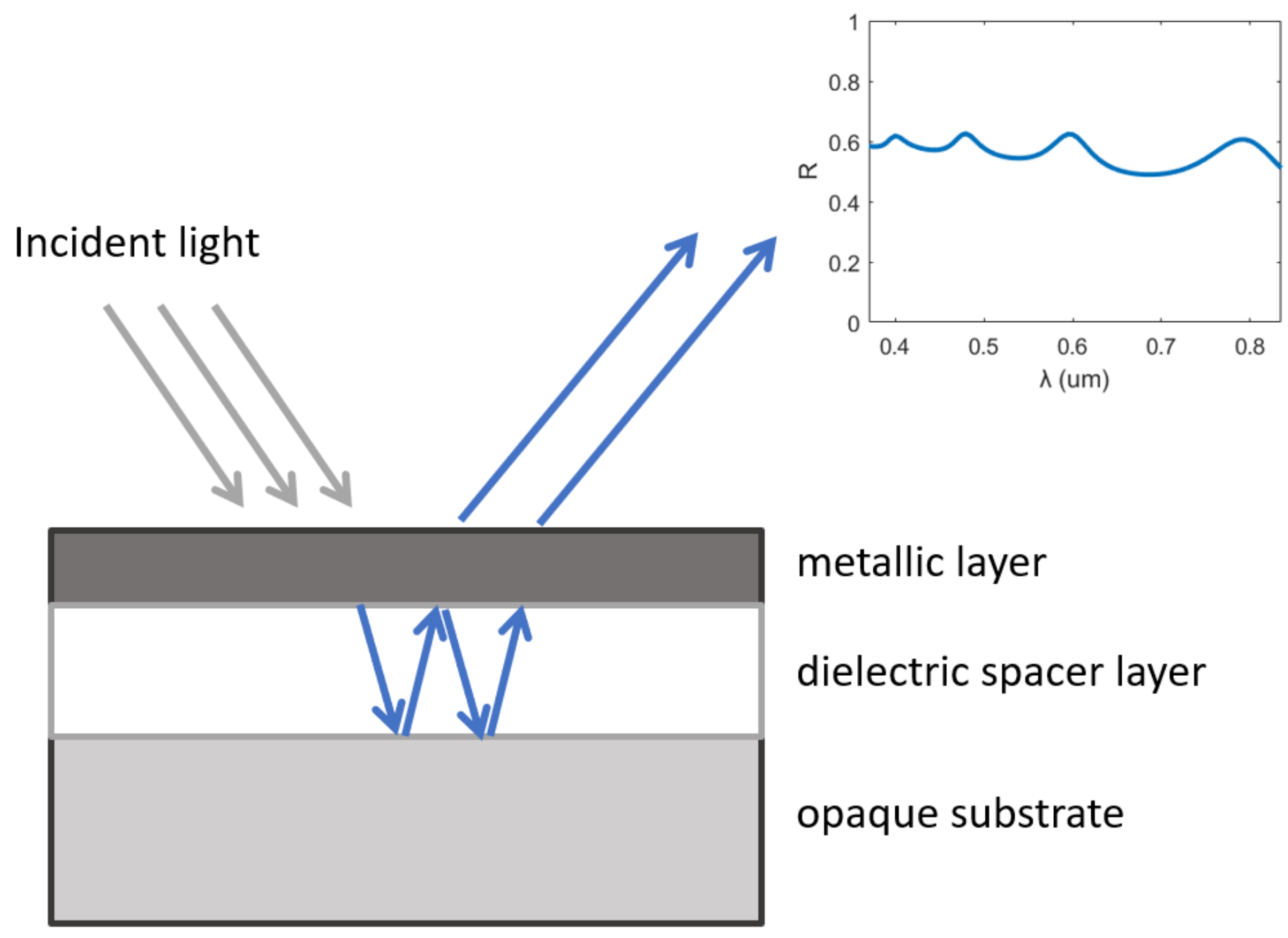

Figure 1

Schematic for the OS (opaque substrate) stack design. Inset shows the typical shape of the resulting reflectance spectrum. 


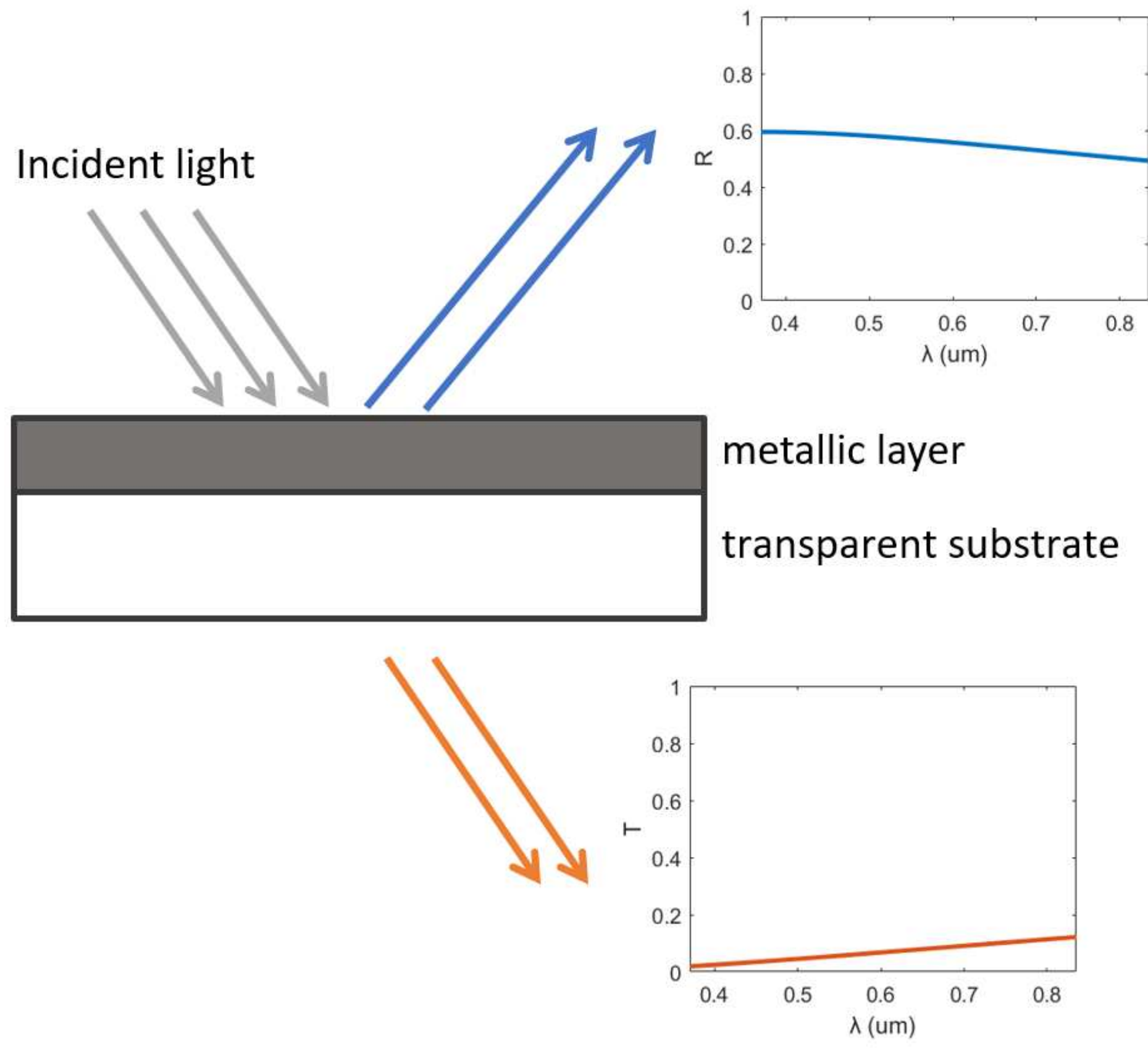

Figure 2

Schematic for the TS (transparent substrate) stack design. Insets show the typical shapes of the resulting reflectance and transmittance spectra. 


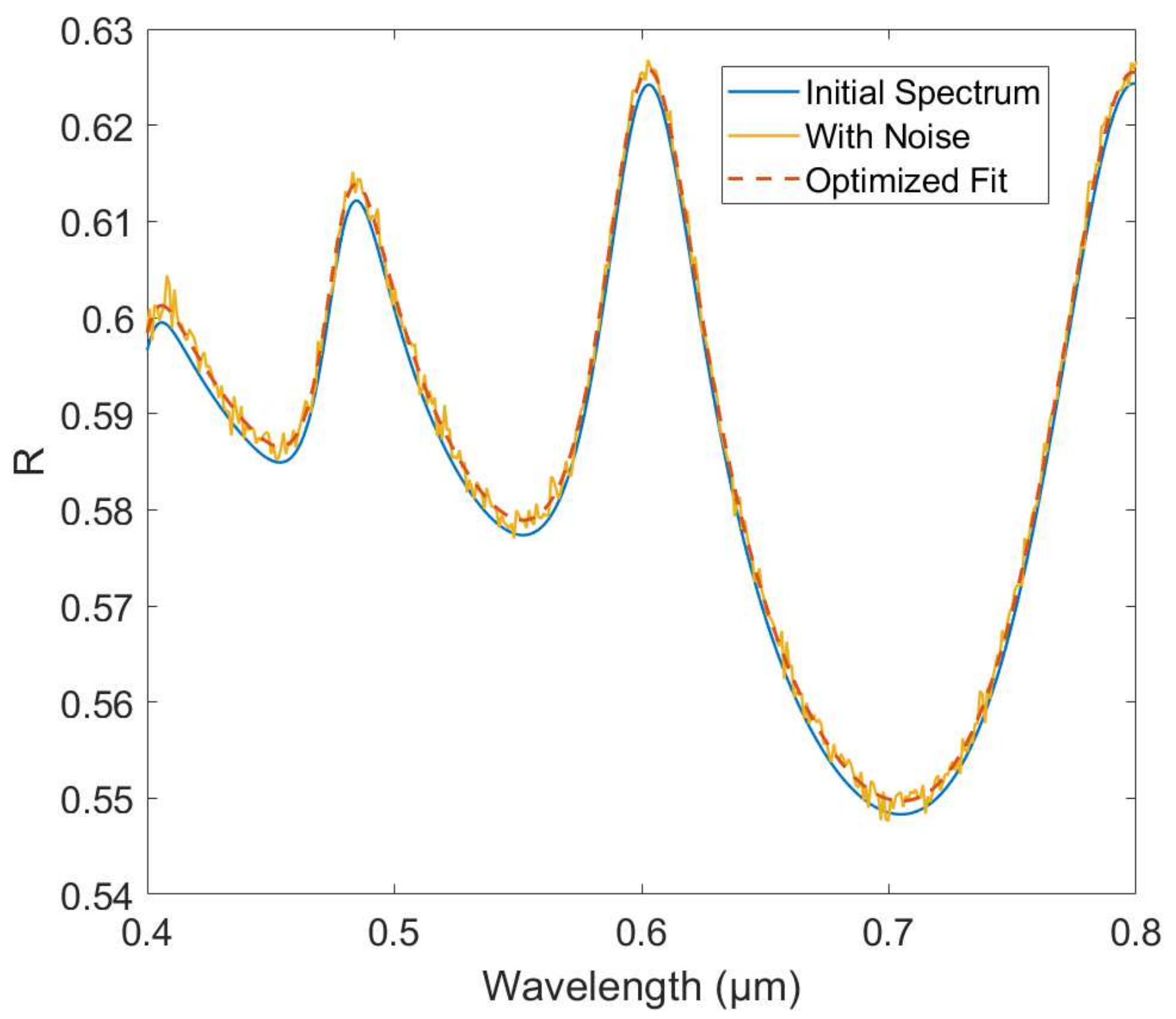

Figure 3

The stages of tting to determine noise resistance: the initial simulated reflectance spectrum (blue), here for the OS stack; a generated noisy spectrum (red); and the resulting fit (purple, dashed). 

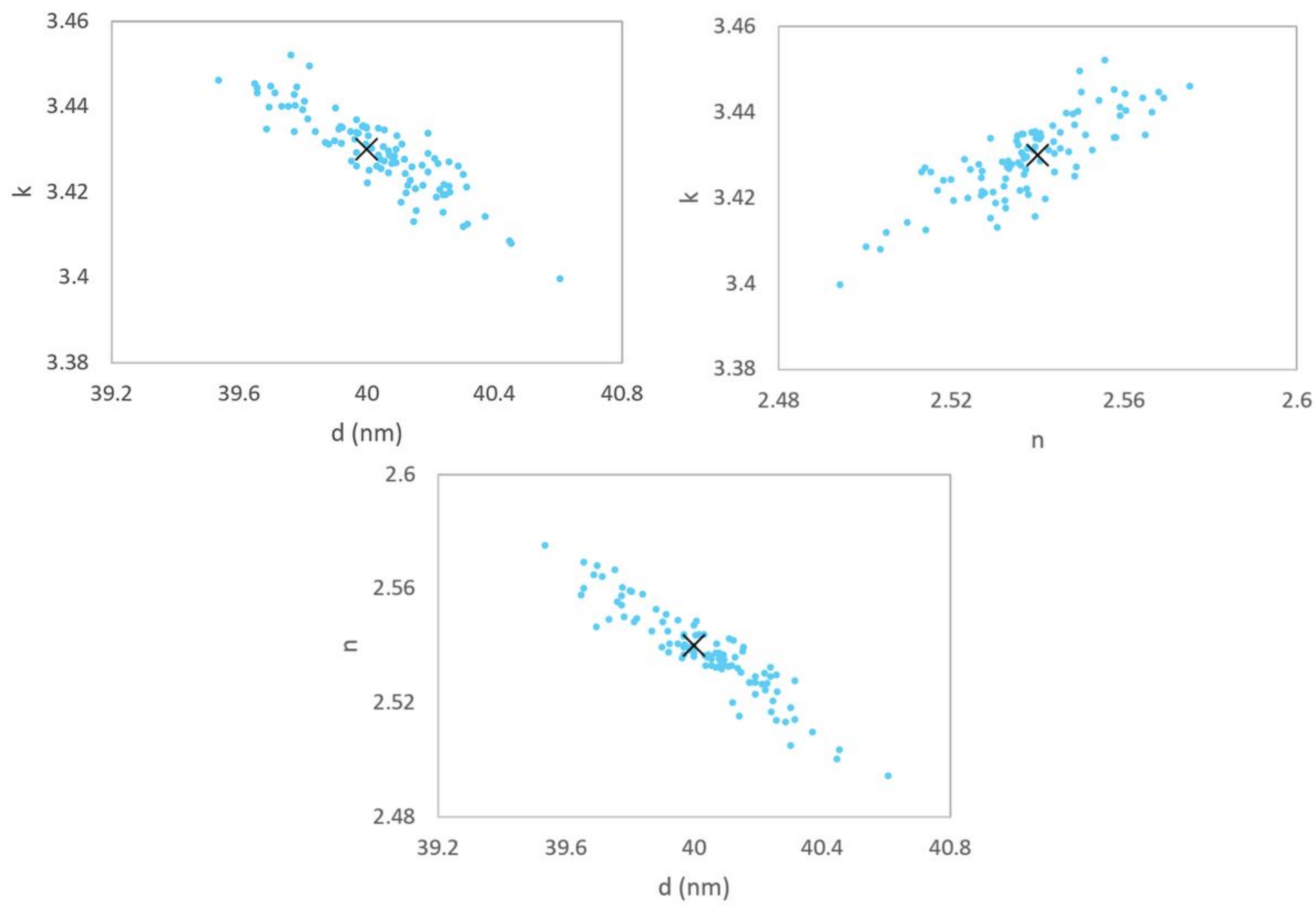

\section{Figure 4}

Three sample scatter plots of $100 \mathrm{n}, \mathrm{k}$ and $\mathrm{d}$ values for the metallic toplayer from tting against spectra with normally distributed additive $(\mu=0, \sigma=0: 001)$ and multiplicative $(\mu=1, \sigma=0: 001)$ noise, for the OS stack with a $800 \mathrm{~nm}$ dielectric layer and a $40 \mathrm{~nm}$ metallic toplayer. Top, $k$ against $\mathrm{n}$; mid, $\mathrm{n}$ against d; bottom, $k$ against $d$. The cross on each plot indicates the nominal values used to generate the initial spectrum of $n=2: 54, k=3: 43$ and $d=40 \mathrm{~nm}$. The standard deviation for these data is 0.015 in $n, 0.0116$ in $\mathrm{k}$ and 0.236 , corresponding to the first row in Table 1. 


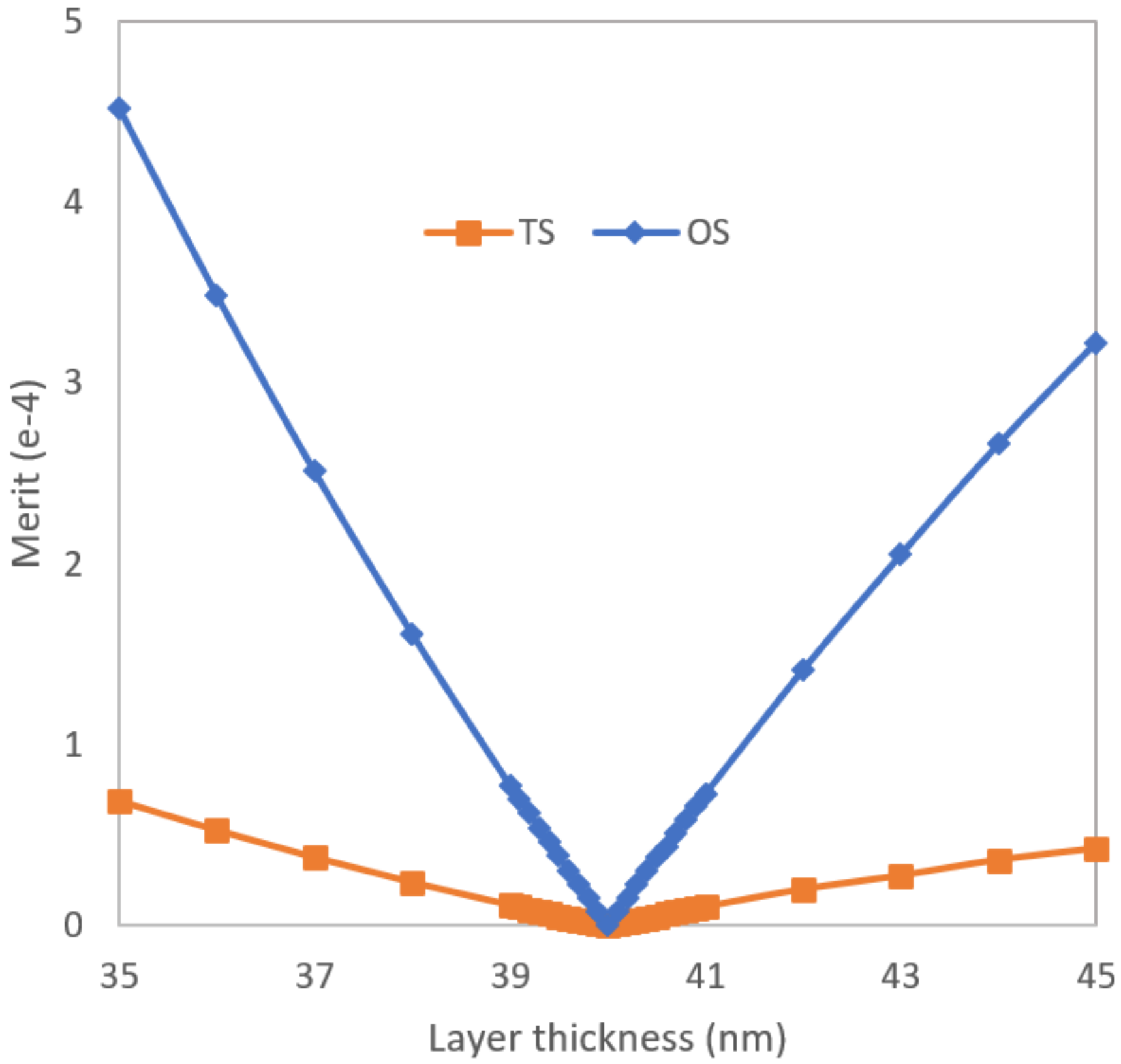

Figure 5

Evolution of least squares merit function with $\mathrm{d}$ fixed and varied around from the optimum at $40 \mathrm{~nm}$, for the two fitting cases (OS and TS). 


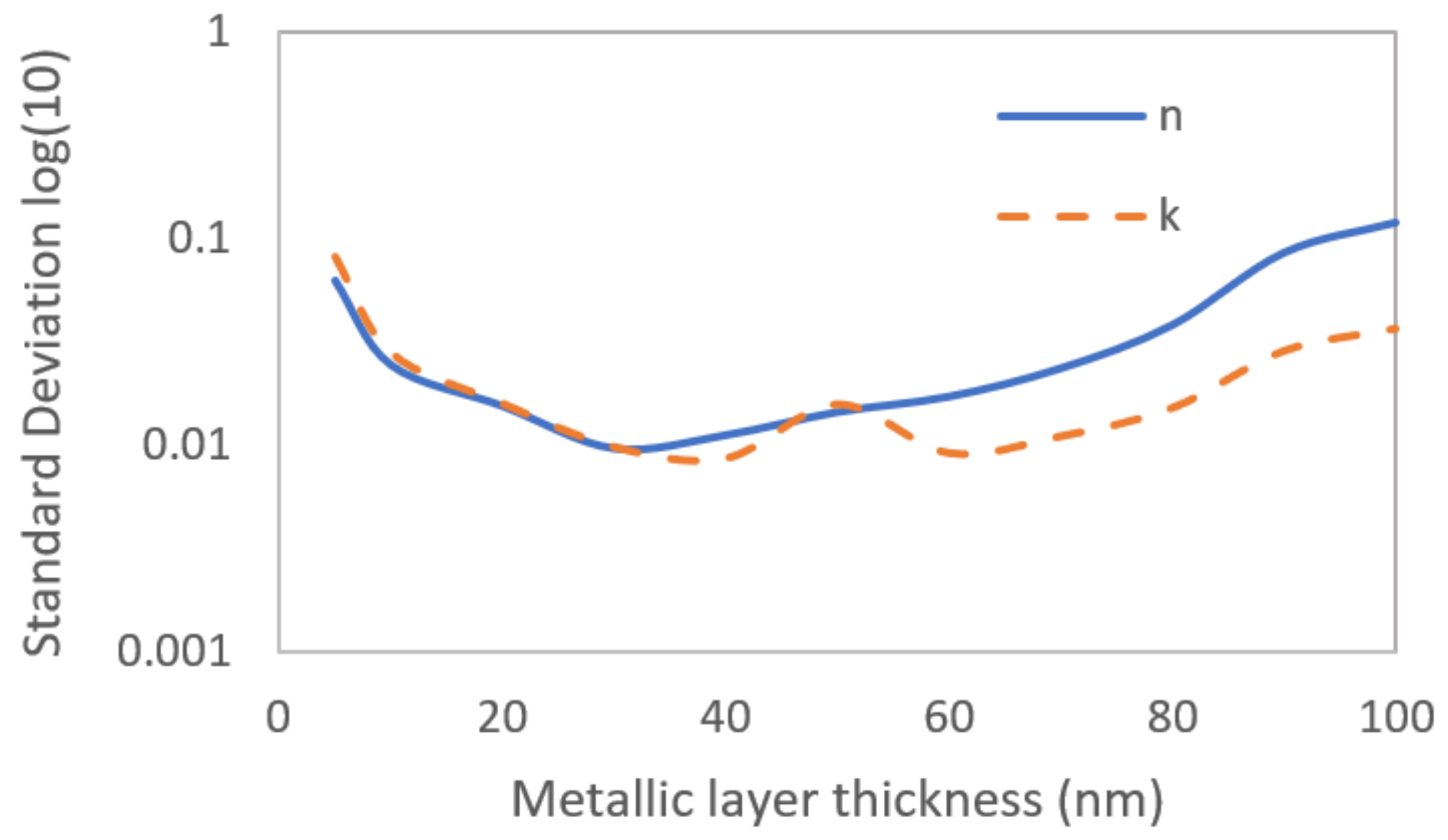

Figure 6

Evolution of standard deviation of determined $\mathrm{n}$ (solid) and k (dashed) for different simulated thicknesses of the metallic toplayer. 


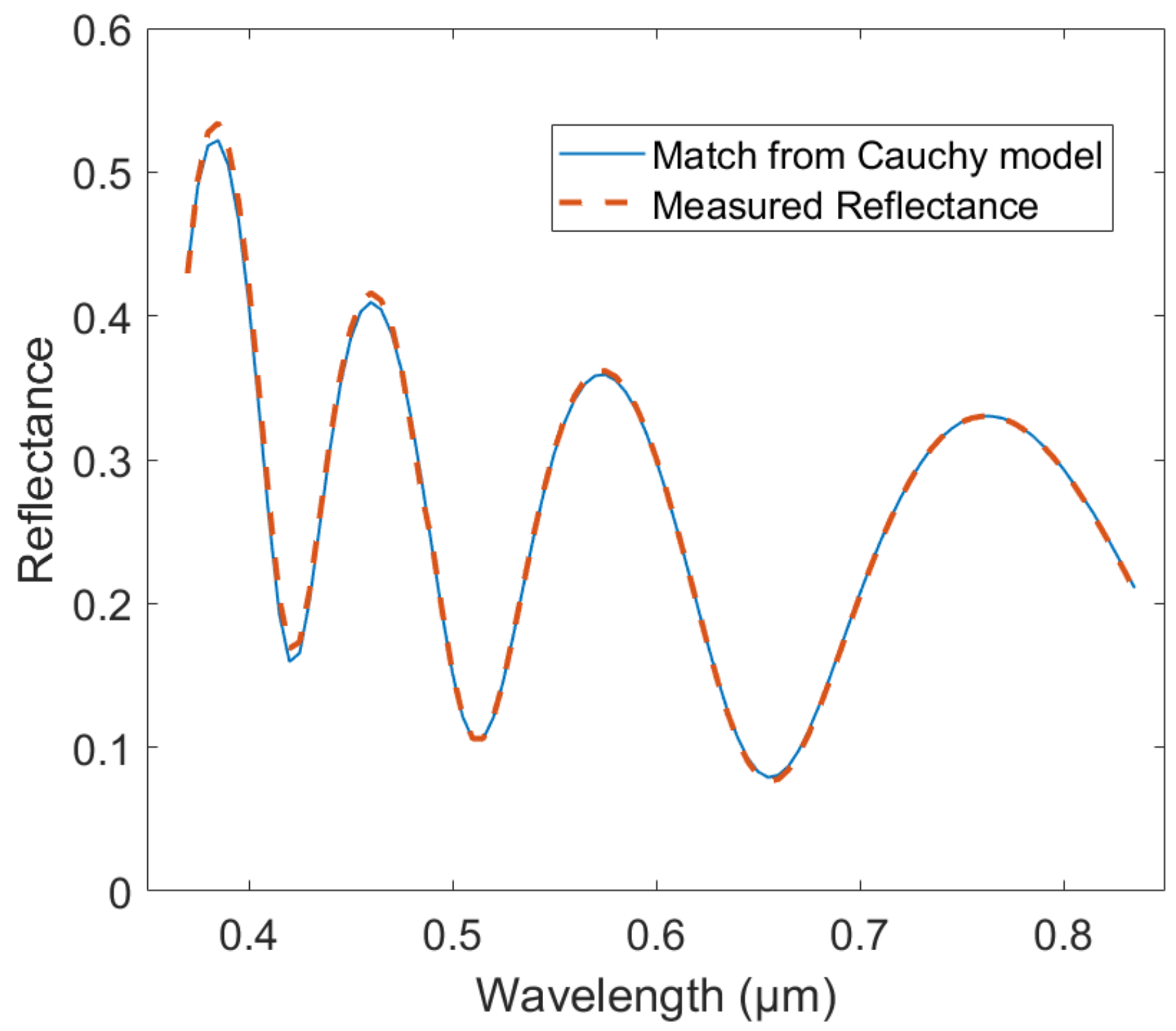

Figure 7

Measured reflectance spectrum from Si substrate coated with $800 \mathrm{~nm}$ SiO2 (dashed) at near-normal incidence, and the resulting $\mathrm{t}$ for the index of the $\mathrm{SiO} 2$ layer using a two-parameter Cauchy approximation (solid). 

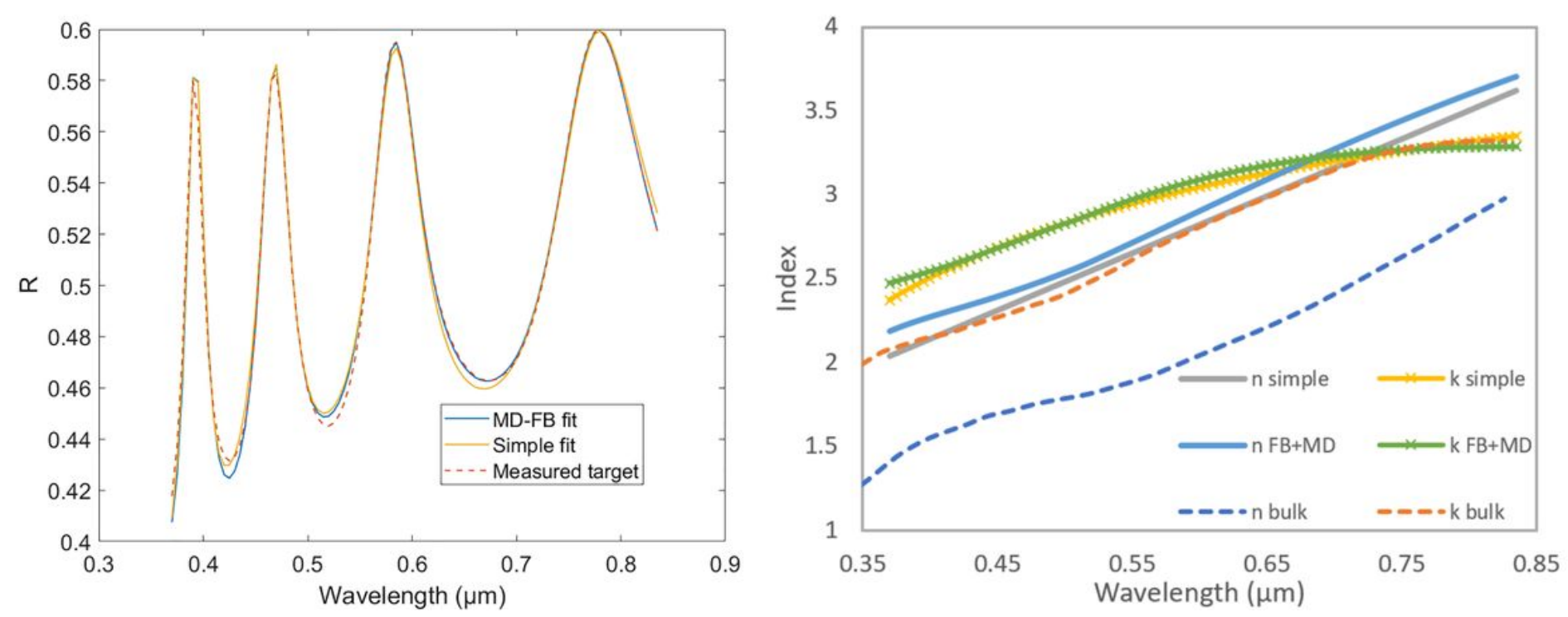

Figure 8

Top: measured reflectance spectrum for $\mathrm{Si} / 800 \mathrm{~nm} \mathrm{SiO} 2 / 28 \mathrm{~nm}$ Ti stack at near-normal incidence, and resulting ts for index dispersion of the Ti layer using simple and MD-FB models. Bottom: comparison between literature data for bulk $\mathrm{Ti}$ (dashed), and simple and MD-FB models for index dispersion of $28 \mathrm{~nm}$ Ti layer (solid).

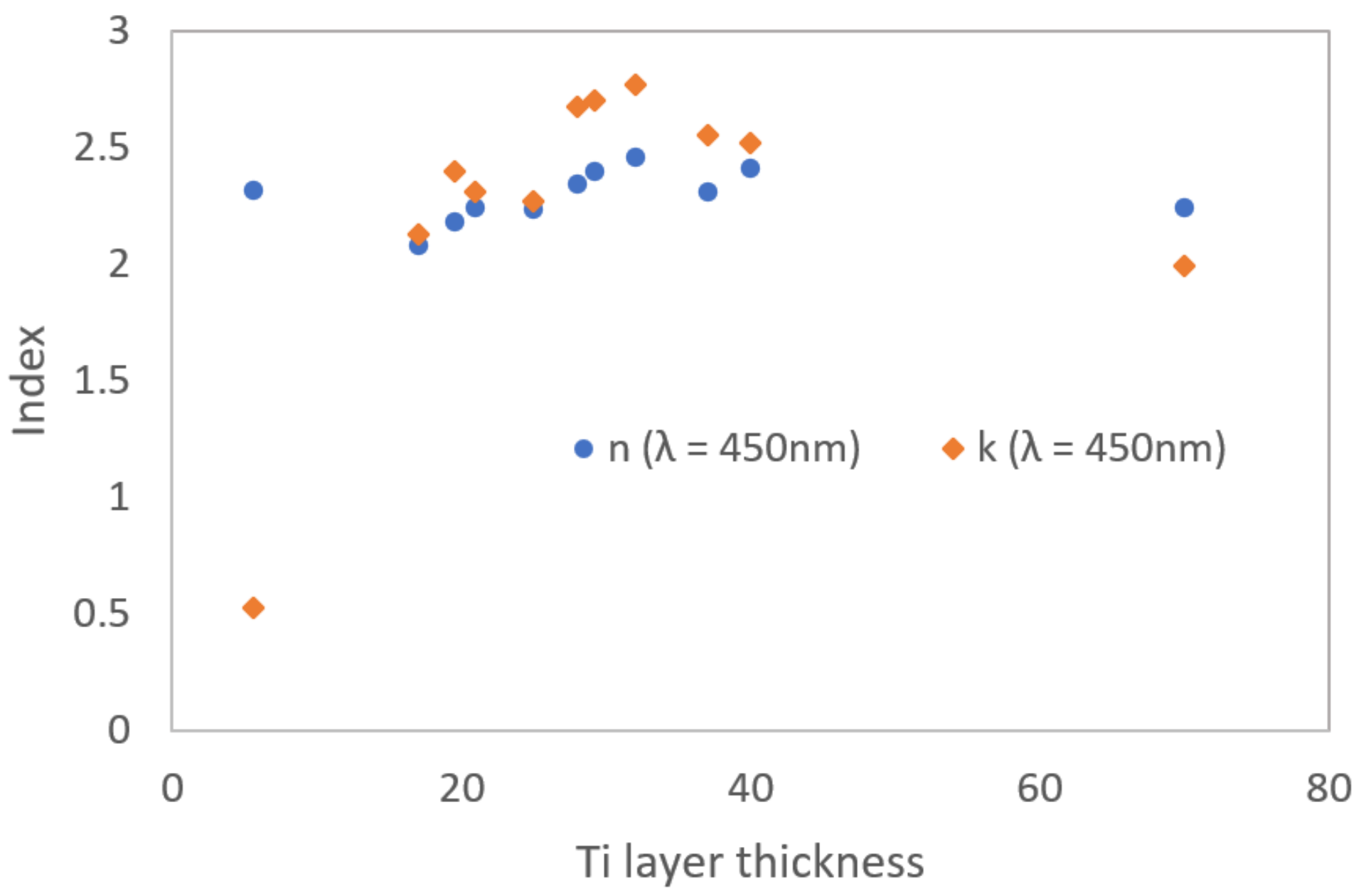




\section{Figure 9}

Evolution of determined $\mathrm{n}$ and $\mathrm{k}$ for different thicknesses of a Ti layer, deposited on top of an Si substrate coated with $800 \mathrm{~nm} \mathrm{SiO2,} \mathrm{shown} \mathrm{at} \mathrm{a} \mathrm{single} \mathrm{representative} \mathrm{wavelength} \mathrm{of} 450 \mathrm{~nm}$. 